\title{
The implement of Internet of things in the College building energy saving design
}

\author{
Chen Jie ${ }^{1, \text { a }}$, Du Aimei ${ }^{1, \mathrm{~b}}$ and Xu Tiecheng ${ }^{1, \mathrm{c}}$ \\ ${ }^{1}$ China architecture design institute co.ltd, No.19, Chegongzhuang Street, \\ Xicheng district, Beijing, China, 100044

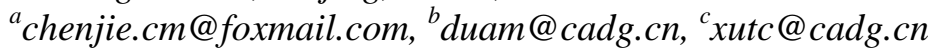

\begin{abstract}
.
This paper introduces the Internet of Things (IoT) technology into building energy-saving design as a novel idea, and considers how to make the new technology play a greater role in building energy saving in campus. The system architecture scheme was introduced to the essential and critical layer of the IoT operating mechanism, called energy sub-metering system and intelligent equipment control system, to realize the intelligent optimization for the management system and the physical environment in college building group. This paper also makes building energy conservation programs with long-term vision in order to avoid the cost increases that caused by the pursuit of high-tech equipment deployment blindly, and then put forward the innovative design against to the building group and the separate area of one building. The conclusion is obviously that the new IoT technology will lead a new direction for building energy saving design that greatly increase the spatial multiplexing possibilities and flexibility, and improve the efficiency of the unit area as well.

Keywords: Internet of Things, College building, Green building, Energy saving

\section{Introduction}

Our college related population which has accounted for $17 \%$ of the total population in urban society, which generates huge energy consumption with their arising use of varied building and territory. Meanwhile, the old campus were kept on going to rebuild due to its limited available space, and the cluster generation of new campus that far from downtown is happened at the same time with the rapid
\end{abstract}


expansion of our city, all of these process constitute a series of energy consumption, therefore contains a huge space of energy saving.

In varied energy-consuming buildings, campus buildings are relatively special groups, which contains infrastructure of community-level just like a miniature city, such as the GYM, library, cafeteria, post offices, teaching buildings, laboratory, etc. Beside these basic functions, whose characteristics are different from the normal public buildings in city, i.e. the scale, direction, rhythm of the crowd movement changes significantly, thus determine the appropriate energy supply and energy consumption characteristics of space, and even the space itself.

In campus, according to the arrangement of semesters and courses, the crowd behavior's distinctive feature is varied with daily, weekly and yearly diversification, meanwhile the crowd activity showing the unique characteristics of centralizing, rhythmic and pulsing. If it is possible to draw a carbon distribution chart with normalized unit of measurement for campus, we'll find that it is the chart of crowd distribution and movement. So we can not ignore the analysis and judgment of human activity characteristics when we performing the building energy saving analysis in campus. This kind of research is seldom in recent years but is important for an architect, and the new technology such as IoT will be the essential way for us.

\section{The introduction of concept of IoT into the energy saving design of college building}

IoT boosts the intellectualization of building. The concept of IoT is using radio frequency identification (RFID), infrared sensors, global positioning systems, laser scanners and other information sensing device to connect any physical entity into Internet according to the agreed protocol, performing information exchange and communication to achieve intelligent identification, positioning, tracking, monitoring and management of the whole network.

Under the situation of advocating sustainable development and low-carbon city, the significance of IoT focuses on promoting intellectualization of buildings, namely the intelligent building is kind of green building with more comfortable 
and more energy saving. The disadvantage of the original building intelligent systems is unable to integrate all sections of varied automatic system, which is overcome by IoT system through integration and upgrade in information sensing, transmission, storage, processing and feedback. The technology of IoT changes the building group into a large closed-loop control system, and changes the energy efficiency management from a relatively extensive way to sensitive and specific way. Thus, building intellectualization combines with IoT will effectively promote the practicing of green building.

The typical IoT application for energy saving in college building. How to embed IoT tech into college buildings depends closely on campus building energy-using characteristics, make it have a direct relationship with crowd mobility features. After summarizing and analyzing varied types of energy consumption preliminary, we found that the electricity consumption is one of the most instable with fluctuation, and also the best indicator of the intensity of crowd activities in campus. In this case, the significance of intelligent monitoring to energy is more inclined to the power monitoring, and which has obvious space for optimization. Below, the basic ideas and methods will be presented briefly on how to embed IoT technology into the energy saving in the electricity system.

Establish of the energy sub-metering system. In the early stage of system design, we should find out the status and regular pattern of energy consumption in college, and then separate them into specific parts to do statistic hierarchically. For example, the information rooms with special electricity system need to be powered all-day and there is no need for sub-metering. In addition, the power consumption of some administrative offices with routine lighting are varied with working days period, we just need to measure it according to the typical week and typical month, then convert them into data format that compliance with the measurement system ultimately, thus we can achieve a better effect of breakdown metering along with the energy characteristics of the system. In contrast, some venues such as teaching laboratory building that with complex and non-regular 
energy consumption, we should install the sub-metering equipment in order to obtain the accurate energy consumption data.

One of the energy sub-metering system called Internet of Building Energy System (iBES) was introduced in [1], that's indeed a kind of IoT application that based on the online awareness of varied types of physical value in building energy system, and it realizes monitoring, control and management of building energy systems through the integration of heterogeneous networks, information aggregation, decision-making diagnosis and feedback control. Especially, the feedback control system show its performance in two aspects, one is the small loop feedback control of internal perceived control layer, the other is the big loop feedback control to the monitoring data after decision-making diagnosis by data center. In the campus energy-saving applications, we could combine above two methods directly include single building and campus building group, which contribute to construct a more integral power monitoring system with distinct-layer.

Intelligent control of electrical equipments. There is also a demo of IoT system of building electrical equipment that introduced in [2], which is divided into five parts according to different functions, namely, RFID module, the environment module, equipment module, the room controller and management computer. RFID module is used to identify personnel information; environment module is used to detect environmental information within the space; equipment module is used to collect device status and parameters; environment modules and equipment modules will transfer the gathered information to the room controller of the space via wireless network, and then upload to the management computer via Ethernet. Management computer will stored received information into database, meanwhile, it can also send control commands to the room controller aiming to control all devices.

\section{Inspiration: Embedded the IoT into the period of campus planning and architectural design}


To achieve embedding IoT into the period of campus physical environment design, we should abide by an opening management system design [3]. On the one hand, we should avoid unnecessary waste of energy in soft environment by varied IoT technology. For example, sharing the classroom Curriculum Schedule with the public educational administration system, to unify the on-off management of air conditioning refer to the classroom Curriculum Schedule, thereby avoiding unnecessary energy waste such as booting for nobody or unreached preset temperature, or keeping open when after-school. On the other hand, which is more worthy of our reflection, is that how to use IoT to rebuild the user's habits in campus and to examine the value of the original planned space, we put forward two feasible schemes as below:

Multiple using and replacement of the space among building group. The IoT application would not only bring the campus more low-carbon by the means of energy consumption gathering, monitoring, management, adaptive feedback, but also take the possibility of space using more efficient in campus, and bring us the new ideas while planning and architectural designing.

Undoubtedly the Internet is weakening the building value of entity space of campus, because the transmission and collection of information in the network replace the communication that face to face in the physical space, while the online courses are more popular, all of these make the original school building gradually in a relatively light load state. So the architect should consider more about these changes.

Colleges as places that existing large numbers of people and people are moving impulsively in it, according to this feature, the "guidance of behavior energy-saving and quantify identification system" [4] should be implemented in campus as one viable option. By which, the information of vacant classrooms, meeting rooms, laboratories are gathering to IoT and becoming a scientific and efficient using time list after analyzing and processing. Then the IoT system interacts with students and teachers in campus though wireless devices such as 
mobile phones, such as help people obtain useful information quickly and use the free space directly.

At the same time, in order to effectively coordinate the monitoring information obtained by IoT, the rooms which have the reuse potential should be given a variety of functions, such as temporarily teaching room could be used at different times for community activities, professional exhibitions, and even learning place for residents of surrounding communities, and so on. Meanwhile, the vacant classroom can also be arranged as a place with obviously professional features, creating different professional office scene, supply the freshmen or students about to enter career an immersive sensory experience and educational location [5]. Another place- cafeteria- is also easy to given multiplexed design, large space and enough tables and chairs are available for students' self-study in the non-meal times using and is consider to supply Specific Dining to enhance the utilization of full time.

Flexible layout for separate building area based on IoT information. At the beginning of a new architecture design, some effects such as composite functions, flexible using, intensive space of one place should be fully considered, combining with the advantages of IoT information, to achieve the association and interaction of person to person, person to material, then using the room almost full load and without interruption, namely reach the maximum of space efficiency. At the same time, the IoT information benefit for the arrangement of educational resources effectively, lead to the demands for teaching space will be further reduced in practical, thus this part of the space is released to the public area, especially to the shared space which will undoubtedly become a necessary core highlights in the comprehensive teach building, it can help students meet varied needs of communication, meeting, leisure, etc., to stimulate creativity and inspiration in a relaxed and lively atmosphere. A design demo is illustrated as Fig.1.

\section{Summary}


The application of IoT in energy saving of public buildings has become much wider, and has been continually updated and upgraded. IoT is already from an initial concept to a multi-layer technical system, gradually penetrates into the practice of smart city and green building. Colleges characterized as unique comprehensive functions similar to community, which offer a plenty of space for IoT in energy saving applications. In which, we have to actively introduce scientific and reasonable IoT technology, but also should not ignore the potential possibility from the new things that changing people's lives, and remained a sensitive manner as a user and designer, especially lead the direction of architectural space towards more comfortable, energy-efficient, enduring wisdom.

(a)

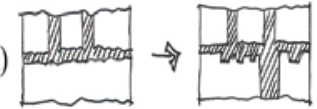

(b)

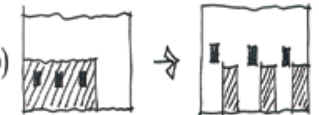

(c)

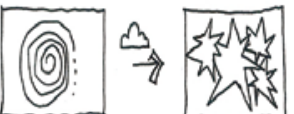

(d)

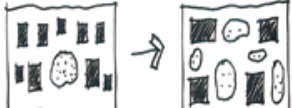

(e)
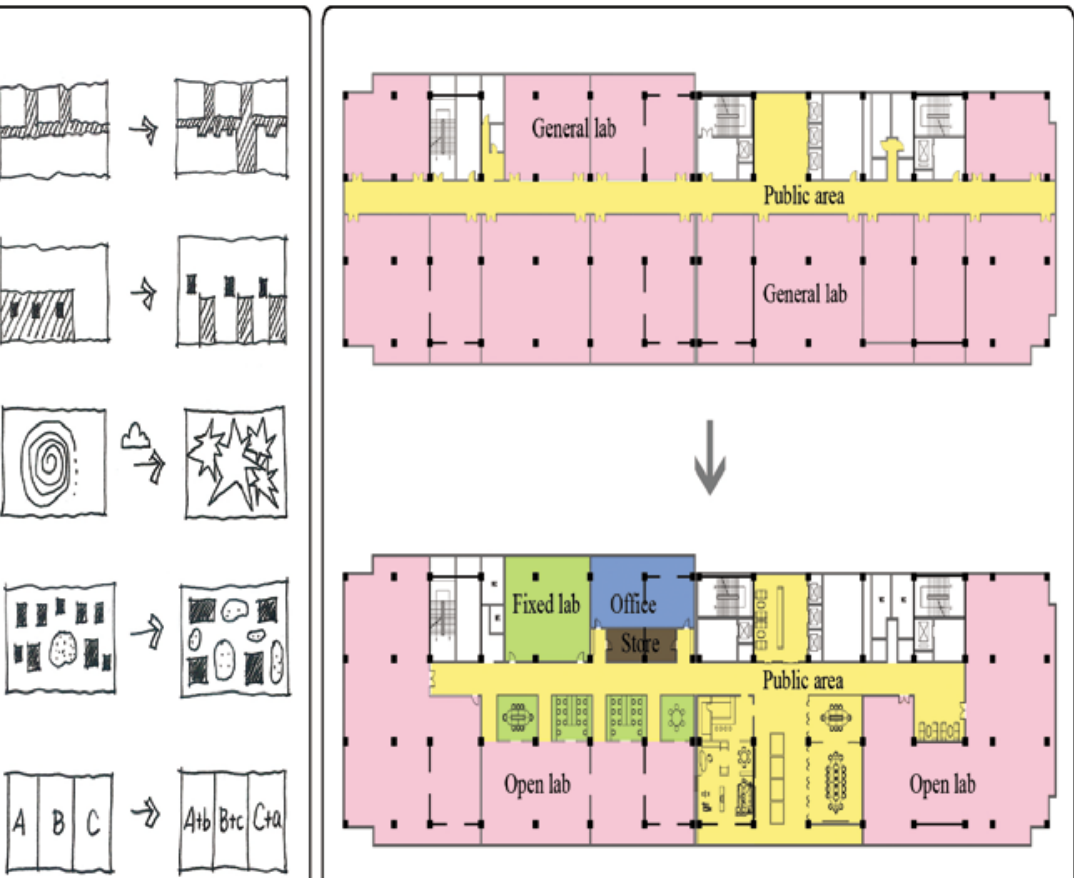

Fig.1. Design demo of flexible layout for separate building area based on IoT information

\section{Acknowledgements}


This work was financially supported by No.15 Architecture Design Studio of China architecture design institute co.ltd.

\section{References}

[1] Chen Yong Pan, in: Research on the framework and technical implementation for the internet of building energy systems, chapter, $3, \mathrm{PhD}$ thesis, Harbin Institute of Technology. (2011)

[2] Shao Zhuhong, in: Research on the Building Electric Equipment Fault Diagnosis and Energy Conservation in the Internet of Things, chapter, 2, Master thesis, Shandong Jianzhu University. (2012)

[3] Wang Ye-zhou: Basic attributes of the Internet of Things and Chinese context. Journal of Nanjing University of Posts and Telecommunications (Social Science), Vol.14, No.2. (2012)

[4] Wang Zhongfeng, Zhang Lianling, Li Ligang, Huang Jianlong: Application research of the internet of things in building energy-saving. Chinese Journal of Scientific Instrument, Vol.31(S2):20-22. (2010)

[5] Tong Wei: Development of Intelligent Building Technology in the Era of Internet of Things. Modern Architecture Electric. Vol.8. (2012) 